\title{
Analysis and Application of MEMS Microshield Line in Millimeter Filters
}

\author{
Yue He, Jingfu Bao*, Yijia Du, Junwen Jiang \\ School of Electronic Engineering, University of Electronic Science and Technology of China, Chengdu 611731, China
}

\begin{abstract}
This paper introduces the conformal mapping method for analyzing the quais-TEM characteristic of MEMS microshield line in detail. The conformal mapping method is an accurately method based on the Schwartz-Christoffel transformation which could invert the complex structure of the microshield line to the conventional plate capacitance. In this paper, the characteristic impedance $Z_{0}$ and the effect permittivity $\varepsilon_{e f f}$ of the microshield line in wide dimension range are obtained. Making use of the simulation result, three filters are designed in millimeter frequency. Furthermore, the S-parameter of the filter indicates the filter designed by the line has good performance, low insertion loss and good out-band suppression.
\end{abstract}

Keywords: Microshield line, millimeter wave, low dispersion

\section{Introduction}

With the development of the communication system recently, the device in the system is required with the feature of low dissipation, small dimension and high performance in millimeter wave band, or higher frequency. But, the conventional coplanar transmission line, such as the microstrip line and the coplanar waveguide, exhibits bad performance in the millimeter wave band, especially in terahertz band. So, the performance of the device designed by the conventional transmission line is limited. In 1991 [1], a new transmission line, the microshield line, was introduced to make up the disadvantage of the convention line in high frequency band. The microshield line has superiority of low dissipation and dispersion in wide frequency range. Hence, the devices designed by the line have a good electrical performance. For investigating the characteristic parameter, in this paper, a conformal mapping method is introduced based on the Schwartz-Christoffel transformation [2]-[4]. Recently, some filters were designed by the coplanar waveguide [5],[6]. Because the conventional coplanar waveguide is similar to the microshield line in structure, so, in this paper, three filters is designed using the similar way to design filters by the former. Making use of the analysis result about the characteristic impedance $Z_{0}$ and the effective permittivity $\varepsilon_{\text {eff }}$, three different filters are presented designed by the line. From the simulation result, the three filters have low insertion loss less than $1 \mathrm{~dB}$ and good out-band suppression.

The structure of the microshield line is shown in Fig. 1. The line consists of the upper wafer, the low wafer and the conductor line supported by a thin membrane (1.4 um $\left.\mathrm{Si}_{3} \mathrm{~N}_{4}\right)$. A cavity exists in the interior of the upper wafer with the metalized wall. Because the line is surrounded by the air, the wave propagation on the line could be treated as a pure TEM mode. It is the reason that the line would have good electromagnetism property in wide frequency range.

\footnotetext{
* Manuscript received July 16, 2012; revised August 25, 2012.

Corresponding author. Tel.: +86-028-61830365; E-mail address: baojingfu@uestc.edu.cn.
} 


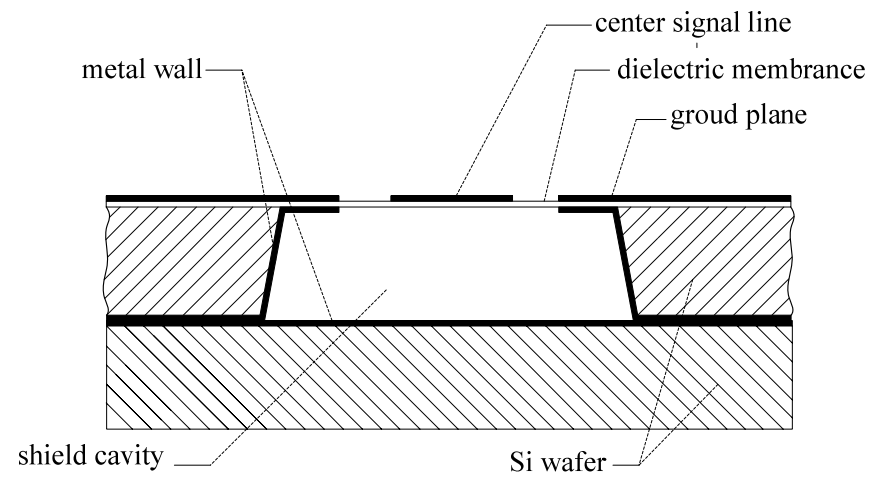

Fig. 1. Structure of the microshield line.

\section{Analysis on the Microshield Line}

\subsection{Introduction about the mapped method}

In order to verify the high performance of the microshield line in millimeter frequency, it is necessary to obtain the characteristic impendence $Z_{0}$ and the relative permittivity $\varepsilon_{e f f}$ of the microshield line. In recently research, the conformal mapping method is an acceptable and accurate analysis method for the derivation of the quasi-TEM parameter of the microshield line. In Fig. 2 (a), the analysis model is presented where there is a rectangular cavity in the upper wafer and overall the metallic conductors are considered to be the ideal conductor to simplify the analysis process. The characteristic impendence $Z_{0}$ of the microshield line is determined by the total capacitance $C_{t}$ per unit length of the microshield line. The total capacitance $C_{t}$ consists of two parts: a) the capacitance of the upper air region and the lower dielectric region. Here, the capacitance of the lower dielectric region is calculated in the conformal mapping method. Firstly, the lower dielectric region is mapping onto the $t$ domain which is one dimensional domain by the Schwartz-Christoffel transformation equation:

$$
z=A \int_{0}^{t}\left(t^{2}-1\right)^{-\frac{1}{2}}\left(t^{2}-t_{c}^{2}\right)^{-\frac{1}{2}} d t
$$

Through this transformation, the simplified two-dimension structure in Fig. 2 (b) is inverted to the one dimension structure in Fig. 2 (c). And in the two figures, the same label indicates the same position in the microshield line. In the conformal mapping method, the upper ground plane of the microshield line extends to the infinite region in the model. Furthermore, the structure of the microshield line is symmetrical, the middle position is considered to the original point in $t$-domain.

Then, the $t$-domain transform back to the $w$-domain which is two-dimensional domain using a second mapping function, as following:

$$
w=\int_{0}^{t} \frac{d t}{\sqrt{\left(t^{2}-t_{a}^{2}\right)\left(t^{2}-t_{b}^{2}\right)}}
$$

where $0<t_{a}<t_{b}<t_{c}<1$.

Through this transformation procedure, the one dimensional $t$-domain turns back to the two dimensional $w$-domain as shown in Fig. 2 (d). In the w-domain, there are two parts: the upper plane represents the center conductor and the lower plane represents the metallic wall of the cavity in the microshield line. So, the two planes form a plate capacitor to be equivalence to the capacitance of the lower dielectric region of the microshield line. And their capacitance values are equivalent. Accordingly, the capacitance value of the microshield line can be simply to the capacitance value of the simple structure plate capacitor by the transformation. 


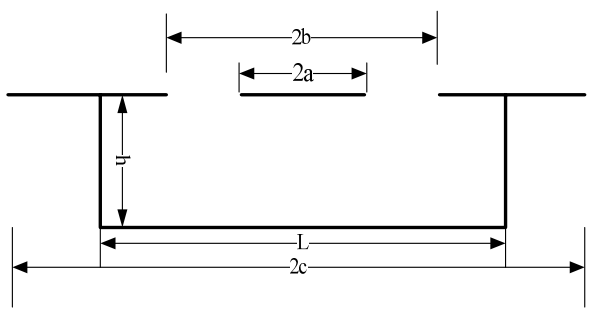

(a)

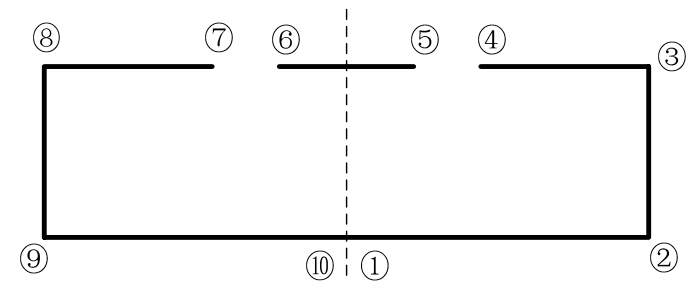

(b)

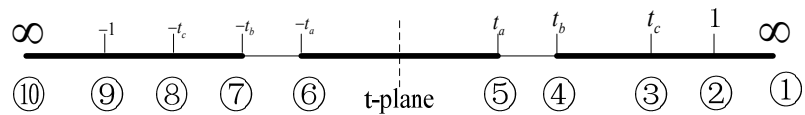

(c)

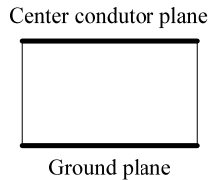

(d)

Fig. 2. Conformal mapping method of microshield line

In the above equation, $t_{a}, t_{b}, t_{c}$ can be calculated in the following expression.

$$
\begin{aligned}
& t_{c}=\left(\frac{e^{\frac{w}{2 h} \pi}-2}{e^{\frac{w}{2 h} \pi}+2}\right)^{2} \quad \text { for } \quad 1<\frac{w}{2 h}<\infty \\
& t_{c}=\sqrt{1-\left(\frac{e^{\frac{w}{2 h} \pi}-2}{e^{\frac{w}{2 h} \pi}+2}\right)^{4}} \text { for } \quad 0<\frac{w}{2 h}<1 \\
& \frac{2 a}{w}=\frac{F\left(\arcsin \left(t_{a} / t_{c}\right), t_{c}\right)}{K\left(t_{c}\right)} \\
& \frac{2 b}{w}=\frac{F\left(\arcsin \left(t_{b} / t_{c}\right), t_{c}\right)}{K\left(t_{c}\right)}
\end{aligned}
$$

where $F(\varphi, \theta)$ is the incomplete elliptic integrals of the first kind, which can be evaluated in MATLAB and MAPLE software. The total capacitance $C_{t}$ of the microshield line is the sum of the upper region and the lower region. If $C_{a}$ and $C_{r}$ refer to the capacitance of the upper region and the lower region respectively, the total capacitance of the microshield line could be calculated by the following equation:

$$
C_{t}=C_{a}+C_{r}=2 \varepsilon_{0} \frac{K\left(k_{1}\right)}{K^{\prime}\left(k_{1}\right)}+2 \varepsilon_{r} \varepsilon_{0} \frac{K\left(k_{2}\right)}{K^{\prime}\left(k_{2}\right)}
$$

where $K\left(k_{2}\right)$ is the complete elliptic integral in the first kind and $K^{\prime}\left(k_{2}\right)$ is the incomplete elliptic integrals of the first kind, $k_{1}=a / b$, and $k_{2}=t_{a} / t_{b}$. Hence, the effective dielectric constant $\varepsilon_{\text {eff }}$ and the characteristic impedance $Z_{0}$ of the microshield line are given by, respectively

$$
\begin{aligned}
& \varepsilon_{e f f}=\frac{C_{t}}{C_{a}+C_{r}\left(\varepsilon_{r}=1\right)} \\
& Z_{0}=\frac{1}{c^{\prime}\left(C_{a}+C_{r}\right) \sqrt{\varepsilon_{e f f}}}
\end{aligned}
$$

Here, $c^{\prime}$ is the speed of light in the air space. For this kind of line, $\varepsilon_{\text {eff }}$ is almost unity. With the help of MATLAB software, the ratio $K\left(k_{2}\right) / K^{\prime}\left(k_{2}\right)$ is easily obtained by the existing function of the elliptic integral. 


\subsection{Simulation result of the conformal mapping method}

The conformal mapping method described in above is an accurate method to analysis the microshield line. Fig. 3 (a) shows the characteristic impedance $Z_{0}$ as a function of the slot width $w$ with the center strip width $s$ as a parameter. As shown, the characteristic impedance $Z_{0}$ of the microshield line has a wide range from 50 to 250. The slot width $w$ and the center width $s$ both have a huge influence on the characteristic impedance. And from the simulation data, once a special characteristic impedance $Z_{0}$ is need, the dimension of the microshield line could be determined. Fig. 3 (b) shows the characteristic impedance as a function of the width ratio $c / b$ with the center $s$ as parameter. As show, the characteristic impedance $Z_{0}$ changes so little when $c / b>2$. It means that the infinite ground plane have negligible effect on the characteristic impedance $Z_{0}$. Because of this, the real ground plane of the microshield line must be enough to reduce the impact that the adjacent lines have interference between them to worsen the performance of the line in a design.

Fig. 4 shows the effective permittivity $\varepsilon_{\text {eff }}$ as a function of the slot width $w$ with the centre conductor width $s$ as a parameter. It is obvious that the effective permittivity of the microshield line is almost unity as the described above. It also confirm that the microshield line have low dissipation and dispersion.

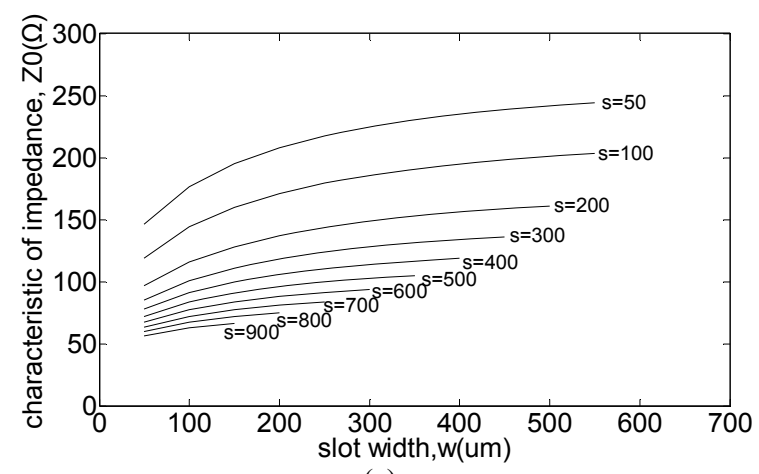

(a)

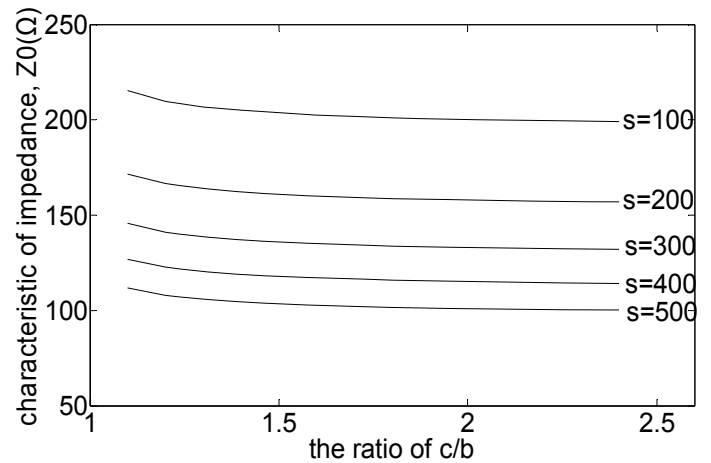

(b)

Fig. 3. Simulation result of the conformal mapping method in MATLAB

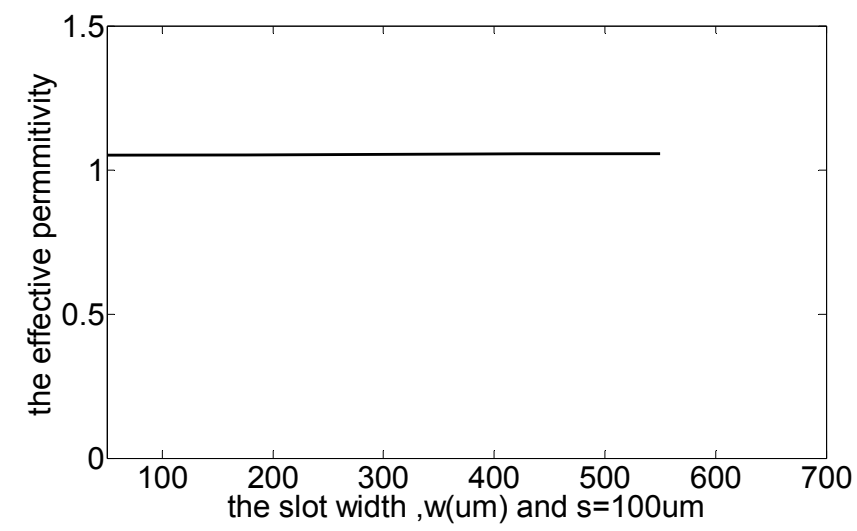

Fig. 4. Simulation result of the effective permittivity $\varepsilon_{e f f}$

\section{Design of Filter in Microshield Line and Simulation Data}

In millimeter wave circuit, low-pass and band-pass filters have great application demands. So, the high performance filter is indispensable device to millimeter wave circuit. The following would introduce three kinds of filter designed by the microshield line to verify the priority of the line on the application in millimeter wave.

\subsection{Low-pass filter}

A low-pass filter designed by the microshield line is a five section $1 \mathrm{~dB}$ equal ripple Cheby-shev filter 
which prototype is shown in Fig. 5. The low impedance and high impedance section of the filter equal to $76 \Omega$ and $265 \Omega$, respectively. The parameter of the filter is initially computed by the normalized initial low pass filter prototype. Then by using the electromagnetism simulation software HFSS, the performance of the stepped low-pass filter could be optimized to get the final filter section lengths. The simulation result of the low-pass filter is shown in Fig. 6. The out-band suppression of the low-pass filter is just close to $20 \mathrm{~dB}$ in $30 \mathrm{GHz}$. But, it is a common method to increase the section of the filter or add a short-stub in output for increasing the out-band suppression of the filter.

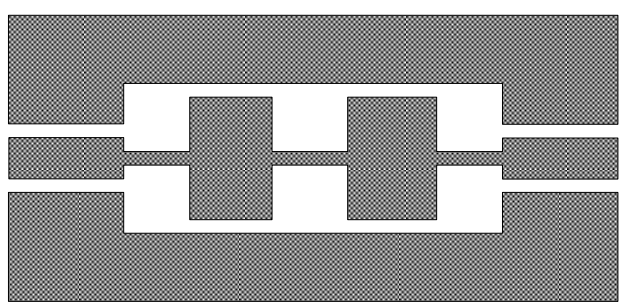

Fig.5. Low-pass filter prototype

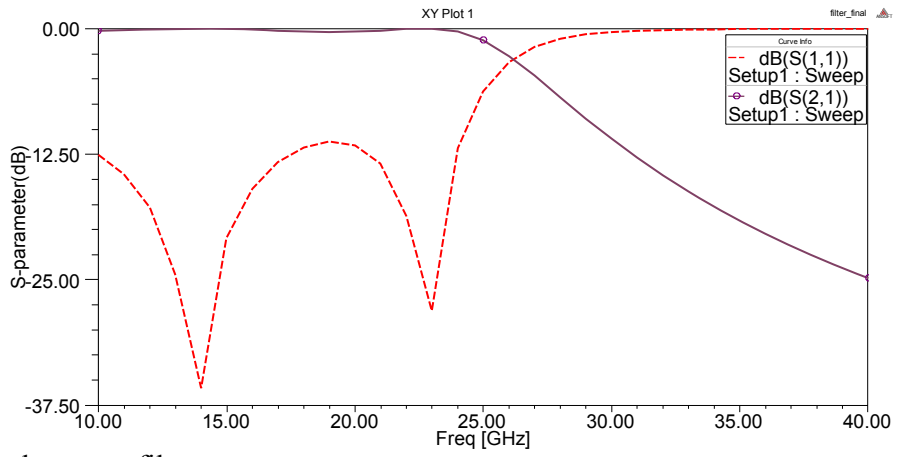

Fig. 6. Simulation result of the low-pass filter

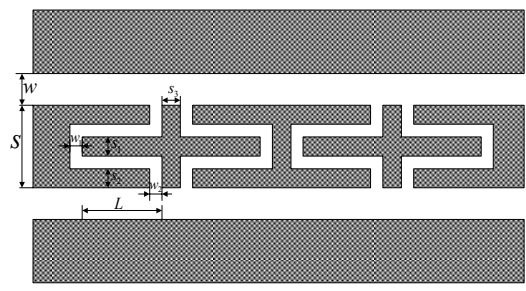

(a)

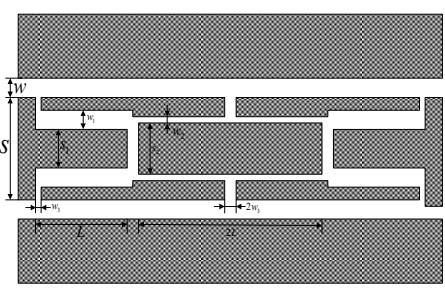

(b)

Fig. 7. Two wide-band band-pass filter prototype

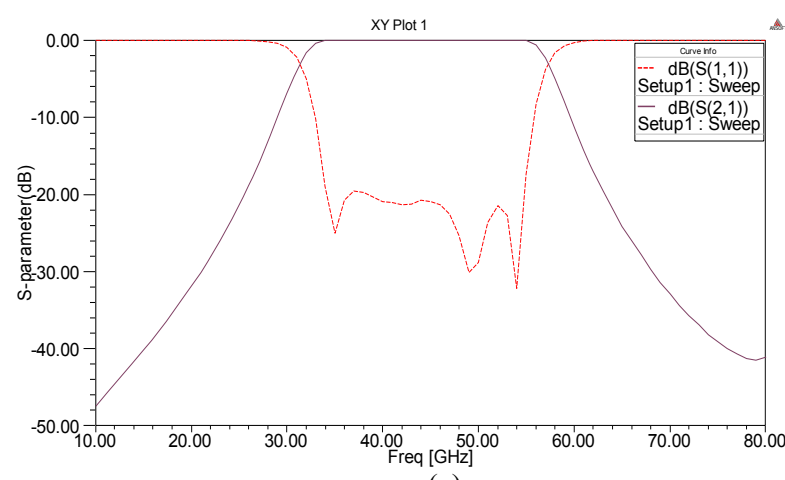

(a)

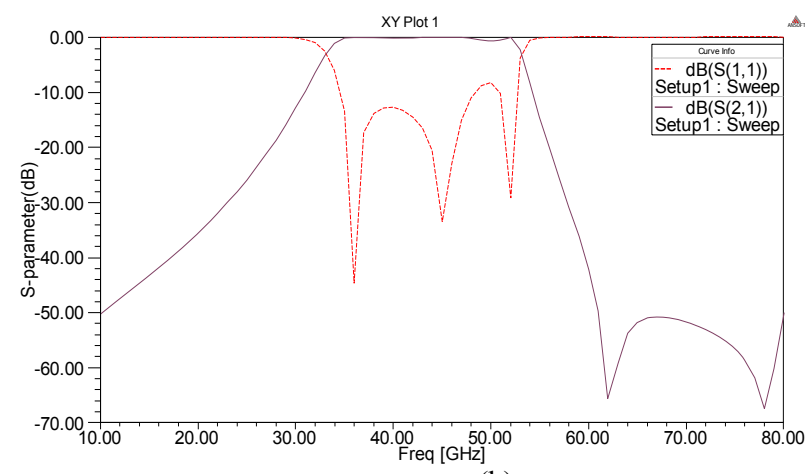

(b)

Fig. 8. The simulation result of two wide-band band-pass filter

\subsection{Band-pass filter}

There are two band-pass filters using the microshield line, but different prototype. The first one is based on the cascaded stubs and another is based on the coupling line of the microshield line. All of them are designed for a centre frequency in $45 \mathrm{GHz}$ and have wide band with almost $50 \%$ relative bandwidth. 
The design way of the two filters is different to the former low-pass filter. In the two filters, the length of the center line is the decisive length which is quarter wavelength. The length determines the center frequency of the filter. These two filters are constructed by the cascaded stubs and the coupling line. So, firstly, the one section stub and the coupling line are simulated in HFSS to obtain the S parameter. Then, four section stubs and the coupling line make up the four sections band-pass filter, as shown in Fig. 7. The simulation results in Fig. 8 show the good performance of the two filters. Both of them have 50\% relative bandwidth and the insertion losses are less than $1 \mathrm{~dB}$ in pass-band.

\section{Conclusion}

The conformal mapping method is the accurate method to evaluate the quasi-TEM characteristic parameter of the microshield line. Through the two times Schwartz-Christoffel transformation, the capacitance of the microshield line could be inverted to the simply plate capacitance. In the paper, the characteristic impedance $Z_{0}$ and the relative permittivity $\varepsilon_{\text {eff }}$ are calculated over a wide range of practical dimensions. It has observed that the slot width and the centre strip width have great effect on the quasiTEM characteristic parameter, respectively. But when the $c / b>2$, the ratio $c / b$ has negligible effect on the characteristic impedance. Three filters designed by the microshield line show good performance on millimeter wave band. From the simulation result, it is obviously that all of the filters have priority on the insertion losses. This is the best proof that the microshield line has low dissipation and dispersion. It could be applied to the millimetre wave circuits and meets the requirement on the rigorous demand in miniaturization and wideband performance of the modem systems. So, the microshield line has better prospect than the conventional coplanar transmission line in the application of the millimeter wave device.

\section{Acknowledgements}

This work was supported by the National Natural Science Foundation of China and the China Academy of Engineering Physics (11176006), the Foundation of China (Grant No. 9140A23060409DZ02)

\section{References}

[1] Dib NI, Harokopus WP, Katehi LPB, Ling CC, Rebeiz GM. Study of a novel planar transmission line. In: Proc. of 1991 IEEE MTT-S Int. Microwave Symp, 1991:623-626.

[2] Cheng KKM, Robertson ID. Quasi-TEM study of microshield lines with practical cavity sidewall profiles. IEEE Trans. on Microwave Theory and Techniques, 1995; 43(12):2689-2694.

[3] Weller TM, Katehi LP. A 250-GHz microshield bandpass filter. IEEE Microwave and Guide Wave Letters, 1995; 5(5):153155.

[4] Ashesh CB, Bhattacharya D, Garg R. Characterization of V-Groove coupled microshield line. IEEE Microwave and Wireless Components Letters, 2005; 15(2):110-112.

[5] Chan KT, Chen CY, Chin A, Hsieh JC, Liu J, Duh TS. 40-GHz coplanar waveguide bandpass filter on silicon substrate. IEEE Microwave and Wireless Components Letters, 2002; 12(11):429-431.

[6] Lin YS, Kao TP, Compact cross-coupled coplanar-waveguide bandpass filter without bondwires, In: Proc. of General Assembly and Scientific Symposium, 2011:1-4. 